\title{
Endobronchial Enchondroma: Unusual Bronchial Tumor
}

\author{
Min Ryu Kyoung ${ }^{1}$, Na-Hye Myong ${ }^{2}$, and Dohhyung Kim ${ }^{1}$ \\ ${ }^{1}$ Dankook University - Cheonan Campus \\ ${ }^{2}$ Dankook University College of Medicine
}

October 24, 2021

\begin{abstract}
Bronchial chondromas are very rare pulmonary benign tumors. Despite their rarity, clinicians should pay attention to the possibility of endobronchial tumor in patients with unexplained respiratory symptoms. Treatment modalities for complete excision of the tumor should be initiated to prevent complications and malignant transformation based on the individual tumor situation,
\end{abstract}

\section{CASE REPORT}

\section{Endobronchial Enchondroma: Unusual Bronchial Tumor}

Kyoung Min Ryu ${ }^{1}$, M.D, Ph.D., Na-Hye Myong², M.D, Ph.D., Dohhyung Kim³ , M.D, Ph.D.

${ }^{1}$ Department of Thoracic and Cardiovascular Surgery, ${ }^{2}$ Department of Pathology, ${ }^{3}$ Division of Pulmonary Medicine and Allergy, Department of Internal Medicine, Dankook University Hospital, Dankook University College of Medicine, Cheonan, Korea

\section{Correspondence}

Dohhyung Kim, Division of Pulmonary Medicine and Allergy,

Department of Internal Medicine,

Dankook University Hospital,

Dankook University College of Medicine, Cheonan, Korea.

Email: kimdh@dankook.ac.kr

\section{Key Clinical Message}

Bronchial chondromas are very rare pulmonary benign tumors. Despite their rarity, clinicians should pay attention to the possibility of endobronchial tumor in patients with unexplained respiratory symptoms. Treatment modalities for complete excision of the tumor should be initiated to prevent complications and malignant transformation based on the individual tumor situation.

\section{KEYWORDS}

Bronchus; Bronchoscopy; Benign tumor; Chondroma; Lung neoplasms

\section{INTRODUCTION}

Even if most endobronchial tumors obstructing bronchi present as bronchogenic carcinoma, benign bronchial tumors can also be a rare cause of bronchial obstruction despite their rarity. ${ }^{1}$ Benign bronchial tumors are usually asymptomatic, and they are found incidentally. However, if they cause bronchial narrowing or 
obstruction, some patients might be suffered from respiratory symptoms according to their complications of bronchi and lungs. ${ }^{2}{ }^{3}$ Here, we present a case of bronchial enchondroma, a very sporadic benign bronchial tumor incidentally found by health screening in a patient who suffered from chronic cough, which was improved after tumor excision.

\section{CASE REPORT}

A 61-year-old man was evaluated in the pulmonary clinic because of a right hilar mass on a simple chest $\mathrm{X}$-ray detected by a national health screening program in South Korea. He was a 30 pack-years current smoker. He had a mild dry cough with scanty sputum persisting for more than six months at his visit, but there was no history of fever, hemoptysis, dyspnea, chest pain, weight loss, night sweats, and other systemic symptoms. He was an office worker, and his past and family histories were unremarkable.

Initial vital signs were stable: Blood pressure, 138/86 $\mathrm{mmHg}$, heart rate, $72 / \mathrm{min}$, respiration rate, 16/min, and temperature, 36.5. There was a slightly reduced breath sound intensity on the right upper posterior chest on chest auscultation, and other systemic physical examinations were normal. A simple chest X-ray showed a well-marginated round mass in the right hilum, which was increased in size compared with the previous chest X-ray taken two years before. (Figure 1) The contrast-enhanced chest computed tomography (CT) was additionally performed, and it revealed about $3.5 \mathrm{~cm}$ sized high-density mass obstructing the right upper posterior segmental bronchus. (Figure 2) The bronchoscopic examination showed a pedunculated, red mass completely obstructing the right upper posterior segmental bronchus. A pinkish, hyaline-like core material with a rigid surface was observed after peeling off the surface mucosa by biopsy procedure. (Figure 3)

In the suspicion of a tumor with the cartilage component, including hamartoma and chondroma, videoassisted thoracoscopic surgery (VATS) was done. The tumor was removed in total and measured $4.0 \times 2.5$ $\mathrm{cm}$ after excision (Figure 4). The histopathology of the excised tumor revealed a bronchial enchondroma composed of lobules of mature hyaline cartilage separated by normal cancellous bone or marrow without adipose tissue and smooth muscle component suggesting hamartoma. (Figure 5) In consideration of the possibility of the Carney triad, no specific findings were observed during gastroscopy, and there was no symptom and sign suggesting paraganglioma by repeated examinations. The patient recovered from surgery, and the dry cough resolved on his visit to the pulmonary clinic one month after tumor removal.

\section{DISCUSSION}

Benign tracheobronchial tumors are sporadic tumors representing only $0.2 \%$ of all pulmonary tumors in the United States. ${ }^{4}$ The two most common types of benign pulmonary tumors are hamartomas and papillomas, and leiomyomas, lipomas, chondromas, and neurogenic tumors can present as less frequent benign neoplasms. ${ }^{1}$ Most benign pulmonary tumors are slow-growing and asymptomatic and often remain undiagnosed before they cause bronchial obstruction or mass effects on surrounding organs. The diagnosis of these tumors is often challenging because the symptoms and signs are non-specific, and a simple chest X-ray fails to present remarkable findings. Thus, they are frequently misdiagnosed as more common causes of chronic cough or dyspnea, such as chronic obstructive bronchitis, asthma, and bronchogenic carcinomas. ${ }^{2,3,5}$ Because most of the symptoms of bronchial tumors are primarily caused by their growth and complications to adjacent structures, early detection and treatment are clinically significant.

Bronchial chondromas are very rare benign tumors arising from bronchial cartilage. ${ }^{1}$ Because they are often misclassified as hamartomas, the most common type of endobronchial neoplasm, the accurate incidence of bronchial chondroma is still unclear yet. ${ }^{1}$ In contrast to hamartomas, endobronchial chondromas have a distinct pathology consisting of calcified/ossified cartilaginous components without adipose tissue, smooth muscle, epithelial, and stromal elements characteristics of hamartomas. ${ }^{6,7}$ Well-differentiated chondrosarcomas can resemble chondromas, ${ }^{4,8}$.they can be differentiated by the presence of tissue invasion or mitosis, ${ }^{9}$ and clinicians should also evaluate the possibility of lung metastasis from other skeletal systems considering higher incidence. 
Bronchial chondromas are occasionally present as a component of the Carney triad characterized by the simultaneous presence of gastric epithelioid leiomyoblastoma, extra-adrenal paragangliomas, and pulmonary chondroma. ${ }^{10}$ In this case, after his tumor was defined as an endobronchial enchondroma, in consideration of the presence of the Carney triad, we performed a gastroscopic examination to evaluate the presence of the gastric leiomyoblastoma. We did complete history-taking and physical examination again to assess symptoms and signs representing paragangliomas. However, there was no evidence of the Carney triad, and the tumor was eventually diagnosed as an isolated endobronchial enchondroma, which is extremely rare among pulmonary chondromas.

Although there is no consensus of the best treatment approach to bronchial chondroma, most treatments were performed by complete excision with bronchoscopy or surgery including VATS or open thoracotomy. The choice of therapy should be considered in each case based on the tumor's operability, size, type, and location. ${ }^{10}$ Even if just a small number of cases were reported, none of the patients died or had severe complications regardless of the treatment options, and recurrence after treatment was very low. There was a report that only one presented recurrence among 23 cases of treated cases between 1945 and $1980 .{ }^{9}$ In addition, there was a report presenting the recurrent tracheal chondroma with the sarcomatous transformation. ${ }^{12,}{ }^{13}$ Considering the low risk of treatment morbidity, mortality, and recurrence, and potential risk of malignant transformation and structural destruction of adjacent organs, prompt and proper treatment options for complete excision should be considered individually based on the tumor situation.

In the current case, when the patient visited the pulmonary clinic for the first time, he had a chronic cough for more than six months, and it was initially considered the effect of cigarette smoking by him. Even if most benign bronchial tumors are asymptomatic, some patients may have symptoms, such as intractable cough or hemoptysis, suggesting chronic airway diseases. ${ }^{2,} 3,14$ After excising the tumor entirely by the lobectomy procedure, his cough disappeared one month after discharge from the hospital. This case is a good example emphasizing the benefit of early recognition and adequate treatment of bronchial tumors to clinicians.

\section{CONCLUSION}

Bronchial chondromas are very rare pulmonary benign tumors. Early recognition and diagnosis are challenging because most benign bronchial tumors are asymptomatic before they grow large enough to obstruct airways or cause complications to adjacent structures. Despite their rarity, clinicians should pay attention to patients suffering from unexplained chronic cough, hemoptysis, dyspnea, and recurrent infection, considering the possibility of having endobronchial neoplasm. Prompt and proper treatment options for complete excision of the tumor should be initiated to prevent complications and malignant transformation based on the individual tumor situation.

\section{ACKNOWLEGEMENT}

None

\section{CONFLICT OF INTEREST}

None declared

\section{AUTHOR CONTRIBUTION}

KR: managed the patient and prepared the manuscript.

NM: examined the pathology and prepared the manuscript.

DK: managed the patient and prepared the manuscript collectively.

\section{ORCID}

Kyoung Min Ryu :https://orcid.org/0000-0001-8461-6010

Na-Hye Myong :https://orcid.org/0000-0001-7181-8334 
Dohhyung Kim :https://orcid.org/0000-0002-3567-4254

\section{REFERENCES}

1. Stevic R, Milenkovic B. Tracheobronchial tumors. J Thorac Dis.2016;8:3401-13.

2. Xu LT, Sun ZF, Li ZJ, Wu LH, Zhang ZY, Yu XQ. Clinical and pathologic characteristics in patients with tracheobronchial tumor: Report of 50 patients. Ann Thorac Surg . 1987;43:276-8.

3. Stevic R, Milenkovic B, Stojsic J, Pesut D, Ercegovac M, Jovanovic D. Clinical and radiological manifestations of primary tracheobronchial tumours: a single centre experience. Ann Acad Med Singapore.2012;41:20511.

4. Gaissert HA, Mark EJ. Tracheobronchial gland tumors. Cancer Control 2006;13:286-94.

5. Wilson RW, Kirejczyk W. Pathological and radiological correlation of endobronchial neoplasms: Part I, benign tumors. Ann Diagn Pathol. 1997;1:31-46.

6. Otani Y., Yoshida I., Kawashima O. Benign tumors of the lung: a 20-year surgical experience. Surg. Today. 1997;27:310-12.

7. Rodriguez FJ, Aubry MC, Tazelaar HD, Slezak J, Carney JA. Pulmonary chondroma: a tumor associated with Carney triad and different from pulmonary hamartoma. Am J Surg Pathol. 2007;31:1844-53.

8. Papagiannis A, Tsakiridis K, Arikas S, Tzamtzis E, Kyrmanidou E, Zaramboukas T. Endobronchial chondroma: a rare benign bronchial neoplasm. Pneumon 2010;23:172-5.

9. Fernandez-Bussy S, Labarca G, Descalzi F, Pires Y, Santos M, Folch E, Majid A. Endobronchial chondromas. Respir Care. 2014;59:e193-6.

10. Carney JA. Carney triad. Front Horm Res. 2013;41:92-110.

11. Teschner M, Gerbatsch KP, Lüllig H. Endobronchial chondroma-bronchoscopic resection or thoracic surgery intervention. Pneumologie. 1998;52:249-53.

12. Salmen US, Halttunen P, Taskinen E, Mattila S. Recurrence and malignant transformation of endotracheal chondroma. Ann Thorac Surg. 1990;49:830-32.

13. Jortay AM, Bisschop P. Chondroma of the trachea. Acta Otorhinolaryngol Belg. 1998;52:247-51.

14. Mahmud T, Nasim Z, Saqib M, Fatima S. Intractable cough due to endobronchial chondroma. Respir Med Case Rep. 2019;29:100968.

\section{Legends for Figures}

Figure 1

A. A right hilar mass (arrow) partially obscured by the right pulmonary trunk is observed.

B. Compared with the chest x-ray taken two years before, the right hilar mass increases in size, and the shape is more clearly exposed.

\section{Figure 2}

The contrast-enhanced chest computed tomography shows about a $3.5 \mathrm{~cm}$ sized well-defined high-density mass (arrow) in the right upper posterior segment, obstructing the posterior segmental bronchus.

Figure 3.

A. The round mass with a red-colored, smooth surface completely obstructing the right upper posterior segmental bronchus is observed by the bronchoscopic examination.

B. Pinkish, hyaline-like core (arrow) is exposed by the biopsy procedure. 


\section{Figure 4}

A big, twisted bronchus is nearly obstructed by a bronchial enchondroma (arrow) composed of lobules of mature hyaline cartilage separated by normal cancellous bone or marrow.

\section{Figure 5}

A. Bronchial enchondroma grows along the bronchial walls, showing diffuse luminal narrowing and multifocally near obstruction (arrowheads).

B. Mature cartilage lobules of this bronchial enchondroma are peripherally encased by bone with endochondral ossification (arrows).

C. and D. Both components of the enchondroma are normal cancellous bone with fatty (C, star) hypercellular (D, star) marrows and mature hyaline cartilage lobules, with no other hamartomatous adipose tissue or smooth muscle components.

\section{Hosted file}

Figures.pptx available at https://authorea.com/users/442637/articles/542853-endobronchialenchondroma-unusual-bronchial-tumor 